\title{
Streptococcus pneumoniae Serotype 5 IgG Antibody Measurement
}

National Cancer Institute

\section{Source}

National Cancer Institute. Streptococcus pneumoniae Serotype 5 Ig G Antibody

Measurement. NCI Thesaurus. Code C142253.

The determination of the amount of Streptococcus pneumoniae serotype $5 \lg G$ antibody present in a sample. 